\title{
Target Tracking Using Reinforcement Learning and Neural Networks
}

\author{
Jezuina Koroveshi and Ana Ktona
}

\begin{abstract}
Target tracking is a process that may find applications in different domains such as video surveillance, robot navigation and human computer interaction. In this work we have considered the problem of tracking a moving object in a multi agent environment. The environment is a rectangular space bounded by walls. The first agent is the target and it moves randomly in the space. The second agent should follow the target, keeping as close as possible without crashing with it. It uses sensors to detect the position of the target. The sensor readings give the distance and the angle from the target. We use reinforcement learning to train the tracker to detect any change in the movement of the target and stay within a certain range from it. Reinforcement learning is a form of machine learning in which the agent learns by interacting with the environment. By doing so, for each action taken, the agent receives a reward from the environment, which is used to determine positive or negative behaviour. The goal of the agent is to maximise the total reward received during the interaction. This form of machine learning has applications in different areas, such as: game solving with the most known game being AlphaGO; robotics, for design of hard-to engineer behaviours; traffic light control, personalized recommendations, etc. The sensor readings may have continuous values, making a very large state space. We approximate the value function using neural networks and use different reward functions for learning the best policy.
\end{abstract}

Index Terms - neural network, object tracking, reinforcement learning.

\section{INTRODUCTION}

Object tracking is an area that has many applications in different domains, some of them being human computer interaction, video surveillance and robot navigation. Recent technological developments have seen a growth in different types of robots built to carry a large number of tasks. Some of the tasks a robot can perform may include rescue operation, disaster relief, patrolling, autonomous navigation, personal assistants, surgical assistant etc. All these tasks may require some form of object tracking, having a target that needs to be followed, for example a personal assistant that follows you carrying your bag. In computer vision, object tracking is related to finding a specific object in different frames that may be used for example in video surveillance. Machine learning has become a very strong tool for solving different types of problems and even surpassing the humans in certain areas. There are many forms of machine learning such as supervised learning, unsupervised learning, semi-supervised

Published on January 10, 2021

Jezuina Koroveshi, Faculty of Natural Sciences, University of Tirana, Albania.

(e-mail: jezuina.koroveshi@ ${ }^{\circledR}$ sshn.edu.al)

Ana Ktona, Faculty of Natural Sciences, University of Tirana, Albania.

(e-mail: ana.ktona@fshn.edu.al) learning, and reinforcement learning. In the supervised learning the outcome for the given input is known, and the machine must learn to map the output to the input. In the unsupervised learning the outcome for the given input is not known. Here the machine classifies the input into groups based on any commonalities that it finds. Semi-supervised learning is a combination of supervised an unsupervised learning that uses both labeled and non-labeled data. Reinforcement learning is a form of machine learning that is based on learning from experience. Here the learner is exposed to some environment, starts making decisions and gets some feedback which gives it information regarding how good or bad was that decision. Based on the feedback, the learner learns which decisions are more favorable. In this paper we are interested in the problem of following a moving object with the intention of staying within some bounds from it. This is related with the task of object identification, but this is out of the scope of this paper. In order to track the target, it will emit some light that will make it recognizable. Both the target and the follower move in two degrees of freedom. Our approach is to use reinforcement learning for solving this problem. Since reinforcement learning requires some form of reward to be designed in order to orient the learner goals, we will try different rewards and will see how they affect the result. Tests are run in a simulated environment.

The remainder of this paper is organized as follows: In part 2 we do a literature review over work done in the related area. Then in part 3 we give a theoretical background on reinforcement learning concepts and ideas. In part 4 we focus more in depth in the algorithms and techniques that are in use. In part 5 we describe the simulation that we have done, the environment and the experiment, and in part 6 give results and conclusions gathered from this work.

\section{LITERATURE REVIEW}

Here we present shortly a review of other works done related to the problem of object tracking in different areas.

In [1] is presented a framework for navigation and target tracking system for a mobile robot. Here is used a combination of low-cost 3D depth and color imaging (Kinect sensor) to replace higher cost imaging systems in order to identify objects that should be tracked, also to identify free space in the space in front of the robot. Fuzzy logic is used to control the movement of the robot for tracking the target. [2] studied the problem of estimating and tracking the motion of a moving target by a team of mobile robots. Each robot has a directional sensor and for that reason more than one robot (sensor) is needed for solving the task. A hierarchical tracking algorithm is used, 
which uses sensor reading in order to get an estimate of the target motion. [4] built a video tracking system for tracking the movements of a robot in the environment. Light is put on top of the mobile robot in order to be able to track it. A camera is placed under the ceiling pointing towards the arena where the robot moves. The movement of the robot is determined by getting the readings of the camera for the position of light that comes from the robot in two consecutive frames. Here is important to make camera calibrations, in order to map image pixel coordinates to floor coordinates. [12] presented a real-time remote-control system for human detection and tracking. In the proposed system is used a Kinect RGB-D camera as a visual sensing device. The remote-control system is implemented on a four-wheel mobile platform with a Robot Operating System (ROS). In order to achieve the human tracking, is used the nearest neighbor search (NNS) algorithm, which searches the nearest detected human position in the previous frame to the current detection result. [8] have presented the first deep learning model that is successful in learning to control policies using reinforcement learning. The model that is used is a convolutional neural network that is trained with a variant of Q-learning. The model takes the input from raw pixel data, and the output is the value function that estimates the future reward. This model is applied to several Atari 2006 games from Arcade Learning Environment and surpasses the human expert on three of them. [5] treated the problem of active object tracking. This is the problem where a tracker takes as input some visual observation, which may be some frame sequences, and based on that produces the output that is the camera control signal. According to [5] conventional methods handle the tracking and camera control separately, which is a very challenging way to tune them jointly and also includes many expensive trial-and-error in real life. To solve these problems, the authors propose a solution that uses reinforcement learning with ConvNet-LSTM as a function approximation to predict the action for the frame. [17] presented a solution to the problem of visual tracking in videos which learns how to predict the bounding box location of the target object in every frame. The tracking problem is considered as a sequential decision-making problem. The solution proposed uses a recurrent convolutional neural network that is trained with reinforcement learning algorithms in order to learn good tracking policies, taking in consideration inter-frame correlation. The network is trainable off-line and this makes it run in faster frame-rates than real-time. This paper develops a new paradigm for solving the problem of visual tracking, by using recurrent neural networks and reinforcement learning in order to exploit temporal correlation in videos. [11] solved the problem of multi object tracking (MOT) using collaborative deep reinforcement learning. Existing methods for MOT use the strategy of tracking-by-detection. The results of these methods rely on the result of the process of detection, which may not be very satisfactory especially in crowded scenes. The solution that is proposed is a deep prediction-decision network, which uses deep reinforcement learning that simultaneously detects and predicts objects. [15] considered the problem of multi object tracking, which is formulated as decision making in Markov Decision Processes. The lifetime of the objects to be tracked is modeled as a MDP, and data association is achieved using reinforcement learning. In [18] is presented a decision controller based on deep reinforcement learning that maximizes long turn tracking performance without supervision. This is applicable in both single object and multi object tracking problems.

\section{REINFORCEMENT LEARNING}

\section{A. General Presentation}

Reinforcement learning is a form of machine learning that is concerned with sequential decision making. The learning agent learns what is the best action to take in each state of the environment, with the purpose of maximizing a numerical reward signal. The agent may not have any knowledge of the environment and it is not told what to do. Instead, it has to learn the best action through interacting with the environment, a process known as trial and error.

A reinforcement learning system may contain four subelements [13]: a policy that defines how the agent behaves at any given time (what action it takes in every state); a reward signal which is sent to the agent from the environment at each time step and is used to define the goal in reinforcement learning. The reward defines what are the good and bad states, and the objective of the agent is to maximize the total reward it receives.; a value function which indicates how good is a state in the long term, taking into consideration the reward for that state and the rewards of states that are likely to follow; a model of the environment, which may be optional, and is used to make predictions about next states and rewards.

Reinforcement learning algorithms estimate value functions (which may be functions of states or functions of state-action pairs) that determine how good is for the agent to be in a certain state (or how good it is to take an action in a state). The general process of RL may be defined as follows:

1. At each time step $t$, the agent is in a state $\mathrm{s}(\mathrm{t})$.

2. The agent choses one of the possible actions in this state, a(t) and applies that action.

3. After applying the action, the agent transitions in a new state $\mathrm{s}(\mathrm{t}+1)$ and gets a numerical reward $\mathrm{r}(\mathrm{t})$ from the environment.

4. If the new state is not terminal, the agent repeats the step 2, otherwise the episode is finished.

The goal of reinforcement learning is to find an optimal policy which tells how to act in each state in order to maximize the return. In order to learn the optimal policy, value functions are used, such as state value and action value.

[13] define three classes for solving the reinforcement learning task: Dynamic Programming, which is based on the Bellman Equation and depends on a perfect model of the environment; Monte Carlo methods that do not need a model of the environment. They can approximate future rewards from experience, but they update the value when the final state is reached.; Temporal difference methods that are a combination between the previous methods. They do not require a model of the environment and the updates are 
done at each step. These methods learn directly from experience.

\section{B. Markov Decision Process}

A reinforcement learning problem can be modeled as a Markov Decision Process (MDP). A MDP is a stochastic process that satisfies the Markov Property. In a finite MDP, the set of states, actions and rewards have a finite number of elements. Formally, a finite MDP can be defined as a tuple:

$$
\mathrm{M}=(\mathrm{S}, \mathrm{A}, \mathrm{P}, \mathrm{R}, \gamma)
$$

where:

$\mathrm{S}$ is the set of states: $\mathrm{S}=(\mathrm{s} 1, \mathrm{~s} 2, \ldots, \mathrm{sn})$.

A is a set of actions: $A=(a 1, a 2, \ldots$, an $)$.

$\gamma \in[0,1]$ is the discount factor.

$\mathrm{P}$ defines the probability of transitions from $\mathrm{s}$ to $\mathrm{s}$ ' when taking action:

$$
\mathrm{P}_{\mathrm{ss}},=\operatorname{Pr}\left\{\mathrm{s}_{\mathrm{t}+1}=\mathrm{s}^{\prime} \mid \mathrm{s}_{\mathrm{t}}=\mathrm{s}, \mathrm{a}_{\mathrm{t}}=\mathrm{a}\right\}
$$

$\mathrm{R}$ defines the reward function for each of the transitions:

$$
\mathrm{R}_{\mathrm{ss}}=\mathrm{E}\left\{\mathrm{r}_{\mathrm{t}+1} \mid \mathrm{s}_{\mathrm{t}}=\mathrm{s}, \mathrm{a}_{\mathrm{t}}=\mathrm{a}, \mathrm{s}_{\mathrm{t}+1}=\mathrm{s}^{\prime}\right\}
$$

The goal of the agent is to maximize the total reward it receives. The agent should maximize the total cumulative reward it receives in the long run, not just the immediate reward [13]. The expected discounted reward is defined as follows by [13]:

$$
\mathrm{G}_{\mathrm{t}}=\mathrm{R}_{\mathrm{t}+1}+\gamma \mathrm{R}_{\mathrm{t}+2}+\gamma^{2} \mathrm{R}_{\mathrm{t}+3}+\ldots=\sum_{k=0}^{\infty} \gamma^{k} R_{t+k+1}
$$

The sequence of states that end up in a terminal state is called an episode. In case the terminal state is reached after a fixed number of states, this is called a finite-horizon task [3]. When the length of a task is not limited by e fixed number, it is called infinite-horizon task [3].

\section{The Policy}

A policy, written as $\pi(\mathrm{s}, \mathrm{a})$, is a function that takes as an argument the state and an action, and returns the probability of taking the action in that state. If the agent is following the policy $\pi$ at time $t$ then $\pi(\mathrm{a} \mid \mathrm{s})$ is the probability that $\mathrm{at}=\mathrm{a}$ if $s t=s[13]$.

\section{Value Function}

The value of a state under s under policy $\pi$ (state-value function for policy $\pi), v_{\pi}(s)$, is the expected return starting from s and following policy $\pi$. It can be defined formally by [13] as:

$\mathrm{v}_{\pi}(\mathrm{s})=\mathrm{E}_{\pi}\left[\mathrm{G}_{\mathrm{t}} \mid \mathrm{S}_{\mathrm{t}}=\mathrm{s}\right]=\mathrm{E}_{\pi}\left[\sum_{k=0}^{\infty} \gamma^{k} R_{t+k+1} \mid \mathrm{S}_{\mathrm{t}}=\mathrm{s}\right]$, for all $\mathrm{s} \in \mathrm{S}$

The value of taking action a in state s under policy $\pi$ (the action-value function for policy $\pi), \mathrm{q}_{\pi}(\mathrm{s}, \mathrm{a})$, is the expected return starting from state $s$, taking action a and then following policy $\pi$. It can be defined formally by [13] as:

$$
\begin{gathered}
\mathrm{q}_{\pi}(\mathrm{s}, \mathrm{a})=\mathrm{E}_{\pi}\left[\mathrm{G}_{\mathrm{t}} \mid \mathrm{S}_{\mathrm{t}}=\mathrm{s}, \mathrm{A}_{\mathrm{t}}=\mathrm{a}\right]=\mathrm{E}_{\pi}\left[\sum_{k=0}^{\infty} \gamma^{k} R_{t+k+1} \mid \mathrm{S}_{\mathrm{t}}=\mathrm{s},\right. \\
\left.\mathrm{A}_{\mathrm{t}}=\mathrm{a}\right], \text { for all } \mathrm{s} \in \mathrm{S}
\end{gathered}
$$

The value functions satisfy the Bellman equation, which expresses the relationship between the value of a state(stateaction) and the values of it next states(state-actions). The Bellman equation for the action-value function is defined as follows:

$$
\mathrm{Q}_{\pi}(\mathrm{s}, \mathrm{a})=\sum_{s^{\prime}} P_{s s^{\prime}}^{a},\left[\mathrm{R}_{\mathrm{ss}}^{\mathrm{a}}+\gamma \sum_{a^{\prime}} \pi\left(\mathrm{s}^{\prime}, \mathrm{a}^{\prime}\right) \mathrm{Q} \pi\left(\mathrm{s}^{\prime}, \mathrm{a}^{\prime}\right)\right]
$$

where $\mathrm{P}$ is the transition probability and $\mathrm{R}$ is the reward for the next state.

Reinforcement learning has an important characteristic, which is the tradeoff between exploration and exploitation. The learner tries to maximize the reward by picking one of the known actions which has the highest reward so far, thus exploiting the already gained knowledge. On the other hand, the learner should explore new actions that might result in a higher reward.

\section{E. On-Policy and Off-Policy Learning}

In [13] on-policy learning is defined as improvement of the same policy that is used to make decisions, while offpolicy is improvement of a policy that is different from the one being used to make decisions. Following that definitions, off-policy methods are more efficient because those can make use of experience replay which allows for usage of samples from different policies.

\section{F. F. Function Approximation}

Function approximation is a method used for generalization when the state and/or action spaces are large or continuous [16]. It generalizes from examples of a function in order to construct an approximation of the entire function. This is a concept related to the supervised learning, studied in the fields of machine learning and pattern recognition [16]. Following is the TD (0) algorithm with function approximation from [13]:

TABLE I: THE TD (0) WITH FUNCTION APPROXIMATION ADAPTED FROM

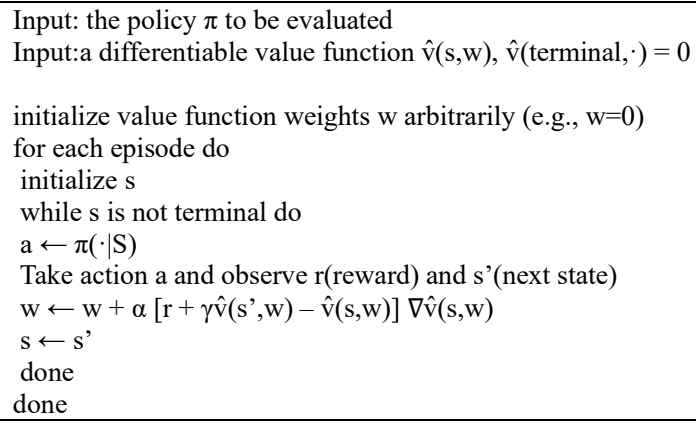

In the above algorithm, $\hat{\mathrm{v}}(\mathrm{s}, \mathrm{w})$ is the approximate value function, $w$ is the value function weight vector, $\nabla \hat{\mathrm{v}}(\mathrm{s}, \mathrm{w})$ is the gradient of the approximate value function with respect to the weight vector [16].

\section{TEMPORAL DIFFERENCE LEARNING}

Temporal difference learning is a central idea in Reinforcement Learning [13]. TD is a combination of dynamic programming and Monte Carlo methods; it learns directly from experience without a model of the environment [13]. TD is a prediction problem, which given 
some experience following a policy $\pi$ it updates the estimate $v \pi$ for nonterminal states St that occur in that experience [13]. In [13] the update rule for TD is defined as follows:

$$
\mathrm{V}\left(\mathrm{S}_{\mathrm{t}}\right) \leftarrow \mathrm{V}\left(\mathrm{S}_{\mathrm{t}}\right)+\alpha\left[\mathrm{R}_{\mathrm{t}+1}+\gamma \mathrm{V}\left(\mathrm{S}_{\mathrm{t}+1}\right)-\mathrm{V}\left(\mathrm{S}_{\mathrm{t}}\right)\right]
$$

where $\alpha$ is the learning rate, and $\mathrm{R}_{\mathrm{t}+1}+\gamma \mathrm{V}\left(\mathrm{S}_{\mathrm{t}+1}\right)-\mathrm{V}\left(\mathrm{S}_{\mathrm{t}}\right)$ is the TD error. This method is called TD (0), which is a special case of $\operatorname{TD}(\lambda)$, because it is based on one step return. Following is the algorithm for TD learning, adapted from [13].

TABLE 2: THE TD ALGORITHM ADAPTED FROM [13]

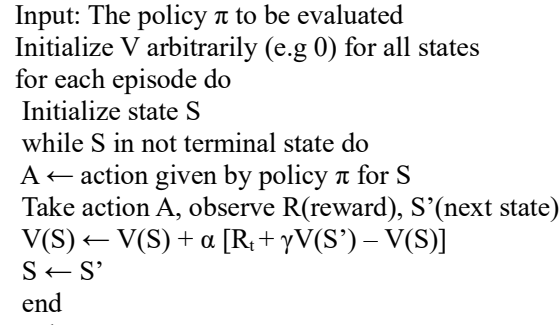

\section{A. On-Policy TD Control with Sarsa}

Sarsa algorithm learns state-action values, instead of state values. As given in [13] it takes into consideration transitions from state-action pair to state-action pair using the following update rule:

$$
\mathrm{Q}\left(\mathrm{S}_{\mathrm{t}}, \mathrm{A}_{\mathrm{t}}\right) \leftarrow \mathrm{Q}\left(\mathrm{S}_{\mathrm{t}}, \mathrm{A}_{\mathrm{t}}\right)+\alpha\left[\mathrm{R}_{\mathrm{t}+1}+\gamma \mathrm{Q}\left(\mathrm{S}_{\mathrm{t}+1}, \mathrm{a}_{\mathrm{t}+1}\right)-\mathrm{Q}\left(\mathrm{S}_{\mathrm{t}}, \mathrm{A}_{\mathrm{t}}\right)\right]
$$

Following is the pseudo code for the Sarsa algorithm.

TABLE 3: SARSA ALGORITHM ADAPTED FROM [13]

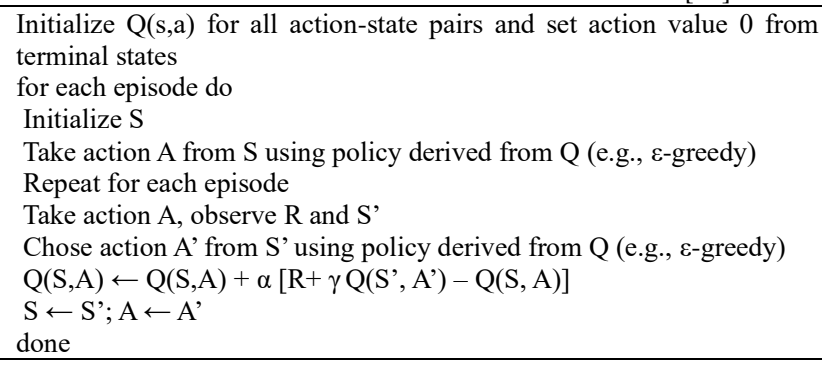

Sarsa is considered an on-policy method, which improves the same policy that it uses to choose the action.

\section{B. Off-Policy TD Control with Q-Learning}

Q-Learning [14] is also considered as a TD method. It is defined with the following update rule:

$$
\begin{gathered}
\mathrm{Q}\left(\mathrm{S}_{\mathrm{t}}, \mathrm{A}_{\mathrm{t}}\right) \leftarrow \mathrm{Q}\left(\mathrm{S}_{\mathrm{t}}, \mathrm{A}_{\mathrm{t}}\right)+\alpha\left[\mathrm{R}_{\mathrm{t}+1}+\gamma \max _{\mathrm{a}} \mathrm{Q}\left(\mathrm{S}_{\mathrm{t}+1}, \mathrm{a}\right)-\right. \\
\left.-\mathrm{Q}\left(\mathrm{S}_{\mathrm{t}}, \mathrm{A}_{\mathrm{t}}\right)\right]
\end{gathered}
$$

In this case, what is learned is the action-value function Q. This is an approximation of the optimal action-value function, independent of the policy that is being followed. Following is the algorithm for Q-Learning, adapted from [13]:
TABLE 4: Q-LEARNING ALGORITHM ADAPTED FROM [13]

Initialize $\mathrm{Q}(\mathrm{s}, \mathrm{a})$ arbitrarily for all action-state pairs, set action vale 0 from terminal states

for each episode do

Initialize $\mathrm{S}$

while $\mathrm{S}$ is not terminal state do

$\mathrm{A} \leftarrow$ action from $\mathrm{S}$ using policy derived from $\mathrm{Q}$ (e.g., $\varepsilon$-greedy)

Take action A, observe R(reward), S'(next state)

$\mathrm{Q}(\mathrm{S}, \mathrm{A}) \leftarrow \mathrm{Q}(\mathrm{S}, \mathrm{A})+\alpha\left[\mathrm{R}+\gamma \max \mathrm{Q}\left(\mathrm{S}^{\prime}, \mathrm{a}\right)-\mathrm{Q}(\mathrm{S}, \mathrm{A})\right]$

$\mathrm{S} \leftarrow \mathrm{S}$

done

done

Q-Learning is considered an off-policy method, because it improves a policy that may be different from the one that is used to choose the action.

\section{Neural Network Implementation Approach}

When the state and action space are discrete, Q-values may be stored in a look-up table: $\mathrm{q}_{\mathrm{i}, \mathrm{j}}=\mathrm{Q}\left(\mathrm{s}_{\mathrm{i}}, \mathrm{a}_{\mathrm{j}}\right)$, for $\mathrm{s}_{\mathrm{i}} \in \mathrm{S}$ and $a_{j} \in A$. If the size of $S$ and $A$ increase considerably, or if $S$ is continuous, it is impossible to visit all the states and to test all actions in reasonable time [2]. For this reason, it is more convenient to use the interpolation capabilities of Artificial Neural Networks (ANN). The ANN would be defined as explained by [2]:

1. Let $\mathrm{n}$ be the dimension of $\mathrm{S}$. A state $\mathrm{s}$ is a vector of components $\mathrm{s}_{1}, \mathrm{~s}_{2}, \ldots \ldots, \mathrm{s}_{\mathrm{n}}$.

2. Let $\mathbf{J}$ be the number of actions A. There are possible two neural implementations:

a) one ANN with $\mathrm{n}$ inputs and $\mathrm{J}$ outputs, where every output represent the Q-value $\mathrm{Q}\left(., \mathrm{a}_{\mathrm{j}}\right), \mathrm{j}=\mathrm{i}$ to $\mathrm{J}$

b) J ANN with one output: one output for action.

Following is the process of using ANN, for the $\mathrm{Q}(0)$ prediction problem [2]:

1. Every state $\mathrm{s}$ is presented as a vector $\mathrm{x}$, with dimension $n$. The ANN calculates an evaluation of $Q\left(x, a_{j}\right), j=1$ to $\mathrm{J}$ (dimension of action space).

2. The action $\mathrm{a}_{\mathrm{j}}$ is chosen, according to the exploration/exploitation policy.

3. New state s', and the reward $r$ are observed.

4. The new state s', is presented as an input to the ANN, and its value is calculated by:

$$
\mathrm{V}\left(\mathrm{s}^{\prime}\right)=\max \mathrm{ANN}_{\mathrm{j}}\left(\mathrm{s}^{\prime}\right), 1<=\mathrm{j}<=\mathrm{J}
$$

5. The new evaluation of $\mathrm{Q}\left(\mathrm{x}, \mathrm{a}_{\mathrm{j}^{*}}\right)$ becomes $\mathrm{r}+\gamma \mathrm{V}\left(\mathrm{s}^{\prime}\right)$. The difference between the new value and the old one is the error committed by ANN and is used to modify the weights.

\section{Neural Fitted Q-Learning}

Neural fitted q-learning introduced by [10] proposes a memory based method to train Q-value functions based on multi-layer perceptron. The basic idea of this method, as explained by [10] is the following: the neural value function is not updated on-line but is updated off-line using a set of transitions gathered from experience. Transitions in the form of (s, a, s') are acquired by interacting with the environment. The state is given as an input to the Qnetwork, and the output is given for each of the possible actions. This structure is very efficient because it allows the computation of the maximum value for each state-action value with only one forward pass of the neural network for any given state. The Q-values are parameterized with a 
neural network $\mathrm{Q}\left(\mathrm{s}, \mathrm{a} ; \theta_{\mathrm{k}}\right)$. The parameters $\theta_{\mathrm{k}}$ are updated by stochastic gradient descent.

\section{E. Deep Q-network}

Deep reinforcement learning (deep RL) is obtained when we use deep neural networks to approximate any of the following components of RL: value function, $\hat{\mathrm{v}}(\mathrm{s} ; \theta)$ or $\mathrm{q}^{\wedge}(\mathrm{s}$, $\mathrm{a} ; \theta)$, policy $\pi(\mathrm{a} \mid \mathrm{s} ; \theta)$, where parameters $\theta$ are the weights of deep neural network [16]. On the other side, shallow RL is obtained when linear models, like linear function or binary trees, are used as function approximation [16].

Deep Q-network was introduced by [7]. It has obtained strong performance playing a variety of ATARI games, learning directly from pixels. Following is the pseudo code for DQN as adapted from [7]:

TABLE 5: DQN ALGORITHM ADAPTED FROM [7]

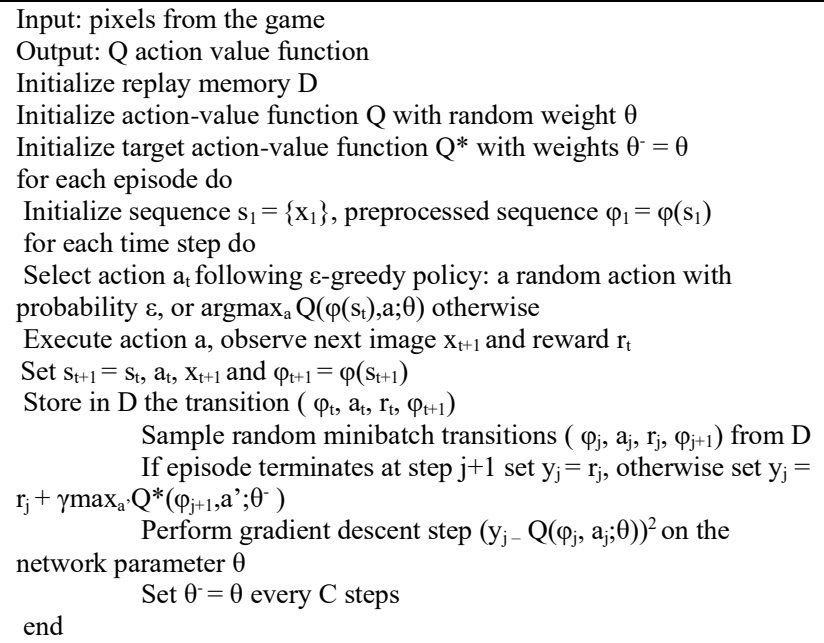

This algorithm has used some heuristics to improve its performance:

1. It uses two networks, $\mathrm{Q}$ and $\mathrm{Q}^{*}$, where $\mathrm{Q}^{*}$ network parameters are updated only every $\mathrm{C}$ iteration. This prevents instabilities from propagating quickly and reduces the risk of divergence.

2. It uses the replay memory. This memory keeps information for the last $\mathrm{N}$ time steps in the form of tuples $<$ s, a, r, s'>, and updates are made on batches selected randomly from the memory. This method allows for updates that cover a wide range of the state action space.

\section{THE PROBLEM}

In this work we have considered a simplified form of the object tracking problem in a multi agent system. The system consists of two agents that move in an empty space that is bounded by walls. Each of the agents can move left, right, or forward. The first agent is the target, and its movements are generated using probability: $1 / 2$ for action forward, $1 / 4$ for action left and $1 / 4$ for action right. This agent emits some light which makes it recognizable in the environment. The second agent should learn to follow the first agent by staying within some bounds form it. It starts from a position behind the first one and has some light sensors that can detect the light emitted from the target. From the light sensed by the sensors he can perceive the position of the target relative to his own. Since both agents can move simultaneously and this makes this task very hard, we have simplified the problem by imposing the following restrictions: both agents move with the same speed; they chose their action one after the other, not moving at the same time. For each action taken from the first agent, the follower should make his move.

\section{A. The Framework Used}

In order to run our experiments, we have used ARGoS Simulator [9], which is a multi-robot simulator for complex environments that involve large swarms of different types of robots. We have used two foot-bot robots for the target and for the follower. Each entity is equipped with sensors that can get information, and with actuators that are used to act in the environment. The target robot has a led actuator that is used to emit red light. The follower robot has omnidirectional camera sensor that can sense light from surrounding objects. We use it to detect red light, since it is the light emitted from the target. The readings of the camera sensor give the angle and the distance from the light. Using these readings, the follower robot can learn the position of the target relative to its own.

\section{B. Proposed Solution}

We have treated this problem as a reinforcement learning problem. For the learner agent, the state consists of two consecutive readings of the camera sensor that give the previous and current position of the target (distance previous, angle $_{\text {previous, }}$ distance current, $_{\text {angle }}$ current), so the state can have continuous values. Based on this, it may choose one of the actions: left, forward, right, no action. We used different types of reward functions that are detailed later in the results section. For doing the training we created e neural network that uses concepts given by [7]. It has a replay memory, and two models. The input to the neural network is the state of the learning agent, and the output is the value for each action. The net has one input layer with 12 neurons, two hidden layers with 12 and 24 neurons, and one output layer with 4 neurons. It uses rectified linear unit as activation function and calculates the error based on mean squared error. The replay memory has a size of 2000 and each replay is done with a batch size of 42 .

\section{Results}

We ran tests with different reward functions and parameters. As a result for each test, we give the number of episodes played and the length of each episode. The length of the episode differs for each test as it is affected by the reward functions and other parameters used. We use the length of the episode to compare the effectiveness of the reward functions. For each test, the reward function, parameters and the number of episodes played is given as follows:

1. If current distance > previous distance or current angle $>$ previous angle then reward $=-1$ and the episode is finished; otherwise, the reward is +1 . The learning rate is $0.8, \Gamma=0.85$ and there are 22000 episodes played. Fig. 1 shows the results.

2. If current distance $>22$ or current distance $<18$ then the reward is -10 and the episode is finished; otherwise, the 
reward is +1 ; The learning rate is $0.01, \Gamma=0.85$ and there are 7500 episodes played. Fig. 2 shows the results.

3 . If current distance $>22$ or current distance $<18$ then the reward is -10 and the episode is finished; otherwise, the reward is +1 . The learning rate is $0.08, \Gamma=0.85$ and there are 8150 episodes played. Fig. 3 shows the results.
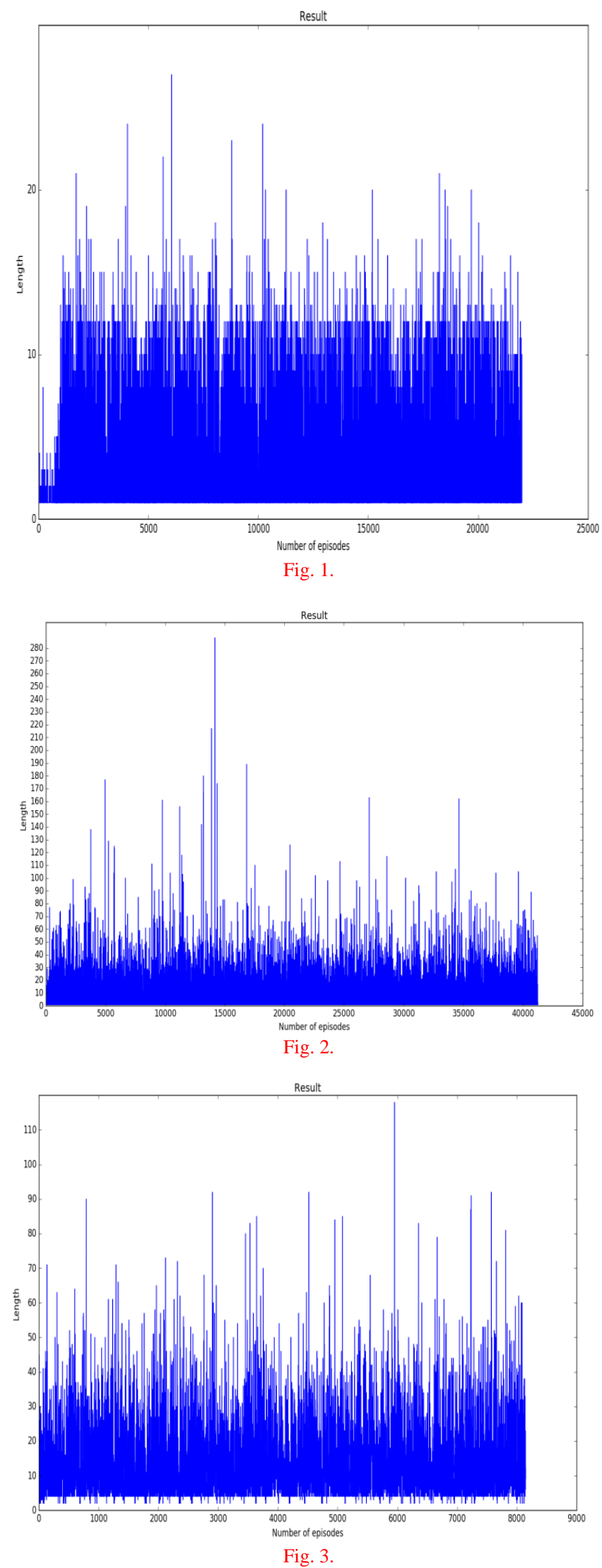

\section{REFERENCES}

[1] Benavidez, P. and Jamshidi, M. (2011) "Mobile robot navigation and target tracking system," 2011 6th International Conference on System of Systems Engineering, Albuquerque, NM, 2011, pp. 299-304. DOI=https://doi.org/10.1109/SYSOSE.2011.5966614.

[2] Glorennec, P. Y. (2000). Reinforcement Learning: An Overview. In Proceedings European Sym. on Intelligent Techniques, (2000).

[3] Kunz, F. (2013). An Introduction to Temporal Difference Learning. Seminar on autonomous learning systems. Department of Computer Science. TU Darmstad.

[4] Lund, H. H., Ves Cuenca, E., Hallman, J. (1996) A Simple RealTime Mobile Robot Tracking System. Techincal Paper no. 41, Department of Artificial Intelligence, University of Edinburgh, 1996.

[5] Luo, W., Sun, P., Zhong, F., Liu, W., Zhang, T., Wang, Y., (2018). End-to-end Active Object Tracking via Reinforcement Learning. In Proceedings of the 35th International Conference on Machine Learning, Stocholm, Sweden, 2018. arXiv:1705.10561 [cs.CV].

[6] Mazo, M., Speranzon, A., Johansson, K. H. and Xiaoming. H (2004) "Multi-robot tracking of a moving object using directional 
sensors," IEEE International Conference on Robotics and Automation, 2004. Proceedings. ICRA '04. 2004, New Orleans, LA, USA, 2004, pp. $1103-1108$ Vol.2. DOI= https://doi.org/10.1109/ROBOT.2004.1307972.

[7] Mnih, V., Kavukcuoglu, K., Silver, D., Rusu, A. A., Veness, J., Bellemare, M. G., Graves, A., Riedmiller, M., Fidjeland, A. K., Ostrovski, G., Petersen, S., Beattie, C., Sadik, A., Antonoglou, I., King, H., Kumaran, D., Wierstra, D., Legg, S. \& Hassabis, D. (2015) Human-level control through deep reinforcement learning. Nature, 518, 529--533. DOI=https://doi.org/10.1038/nature14236.

[8] Mnih, V., Kavukcuoglu, K., Silver, D., Graves, A., Antonoglou, I., Wierstra, D., \& Riedmiller, M.A. (2013). Playing Atari with Deep Reinforcement Learning. arXiv:1312.5602 [cs.LG].

[9] Pinciroli, C., Trianni, V., O’Grady, R., Pini, G., Brutschy, A., Brambilla, M., Mathews, N., Ferrante, E., Di Caro, G., Ducatelle, F., Birattari, M., Gambardella, L. M., Dorigo. M., (2012). ARGoS: a Modular, Parallel, Multi-Engine Simulator for Multi-Robot Systems. Swarm Intelligence, volume 6, number 4, pages 271-295. Springer, Berlin, Germany. DOI=https://doi.org/10.1007/s11721-012-0072-5.

[10] Riedmiller M. (2005) Neural Fitted Q Iteration - First Experiences with a Data Efficient Neural Reinforcement Learning Method. In Gama J., Camacho R., Brazdil P.B., Jorge A.M., Torgo L. (eds) Machine Learning: ECML 2005. ECML 2005. Lecture Notes in Computer Science, vol 3720. Springer, Berlin, Heidelberg. DOI=https://doi.org/10.1007/11564096_32.

[11] Ren, Liangliang \& Lu, Jiwen \& Wang, Zifeng \& Tian, Qi \& Zhou, Jie. (2018). Collaborative Deep Reinforcement Learning for Multiobject Tracking: 15th European Conference, Munich, Germany, September 8-14, 2018, Proceedings, Part III. DOI=https://doi.org/10.1007/978-3-030-01219-9_36.

[12] Sankar S, Tsai C-Y. (2019). ROS-Based Human Detection and Tracking from a Wireless Controlled Mobile Robot Using Kinect. Applied System Innovation. 2019; 2(1):5; DOI=https://doi.org/10.3390/asi2010005.

[13] Sutton, R. S. and Barto, A. G. (2018) Reinforcement Learning: An Introduction (2nd Edition, in preparation). MIT Press.

[14] Watkins, C.J.C.H. and Dayan, P. (1992) Mach Learn 8: 279. DOI=http://dx.doi.org/10.1007/BF00992698.

[15] Xiang, Y., Alahi, A., Savarese, S., (2015). "Learning to Track: Online Multi-object Tracking by Decision Making," 2015 IEEE International Conference on Computer Vision (ICCV), Santiago, 2015, pp. 4705-4713. DOI=https://doi.org/10.1109/ICCV.2015.534.

[16] Y, Li. (2018) Deep Reinforcement Learning: An Overview. arXiv:1701.07274v6 [cs.LG], Nov. 2018.

[17] Zhang, D., Maei, H., Wang, X., \& Wang, Y. (2017). Deep Reinforcement Learning for Visual Object Tracking in Videos. arXiv:1701.08936[cs.CV]

[18] Zhong, Z., Yang, Z., Feng, W., Wu, W., Hu, Y., and Liu, C., (2019). "Decision Controller for Object Tracking With Deep Reinforcement Learning," in IEEE Access, vol. 7, pp. 28069-28079, 2019. DOI=https://doi.org/10.1109/ACCESS.2019.2900476. 